Rapid Reviews COVID-19

\title{
Review 2: "Sensitivity of SARS-CoV-2 antigen- detecting rapid tests for Omicron variant"
}

Monika Klimek-Tulwin ${ }^{1}$

${ }^{1}$ Medical University of Lublin

Published on: Mar 03, 2022

License: Creative Commons Attribution 4.0 International License (CC-BY 4.0). 


\section{RR:C19 Evidence Scale rating by reviewer:}

- Potentially informative. The main claims made are not strongly justified by the methods and data, but may yield some insight. The results and conclusions of the study may resemble those from the hypothetical ideal study, but there is substantial room for doubt. Decision-makers should consider this evidence only with a thorough understanding of its weaknesses, alongside other evidence and theory. Decisionmakers should not consider this actionable, unless the weaknesses are clearly understood and there is other theory and evidence to further support it.

$* * * * * * * * * * * * * * * * * * * * * * * * * * * * * * * * * * * * * * *$

\section{Review:}

In the introduction, the authors rightly pointed out that SARS-CoV-2 antigen-detecting rapid diagnostic tests are characterised by lower sensitivity than RT-PCR.

Nevertheless, due to current public health needs and the need to identify infectious and non-infectious patients, the use of SARS-CoV-2 antigen-detecting rapid diagnostic tests is widely applicable in clinical practice. Unfortunately, with the emergence of new mutations in the genes encoding the virus proteins, the effectiveness of vaccination is decreasing, and the risk of re-infection is increasing. In addition, there is the question of the sensitivity of antigen tests validated with earlier variants of the virus. Has their sensitivity remained constant despite the new coronavirus variants? Researchers are trying to answer this question. No studies are available to date on the sensitivity of AgRDT against specific virus variants that include the Omicron variant hence why this topic is important and valuable.

It is worth noting that only symptomatic patients were included in the study. Thus, we are talking here about sensitivity towards symptomatic patients and not in general, "COVID-19 infected population." Furthermore, the number of samples tested is small, so the results must be interpreted with caution.

The study included as many as eight commercially available Ag-RDT products, which is impressive. Although some of these results have been published before (data concerning analytical sensitivity for Alpha, Beta, Gamma, and Delta variants), in the presented study, these results were presented again to compare them with data 
concerning the Omicron variant. It is worth noting that these earlier papers are the work of the same authors, which increases the reliability of this comparison.

In the results, the authors write, "Two tests showed a slightly higher sensitivity for Omicron than for Delta (Test V and VII)" [V - Beijng Tigsun Diagnostics Co. Ltd (Tigsun); VII - ACON biotech (Flowflex)]. However, the chart shows that it is IV [2019$\mathrm{nCoV}$ Antigen test (Wondfo)] and VII. Was the error in the text or in the graph?

An interesting finding is that overall test positivity for Omicron was much lower when compared to Delta (49.2\% vs. 65.5\%) ( $\mathrm{z}=-3.65, \mathrm{p}<.001)$. Moreover, when comparing sensitivity for Delta vs. Omicron for each Ag-RDT, four Ag-RDTs showed significantly lower sensitivity $(\mathrm{p}<0.001$ ) [Panbio COVID-19 Ag Rapid test device (Abbott); Standard Q COVID-19 Ag (SD Biosensor/Roche); Sure Status (Premier Medical Corporation); Onsite COVID-19 Ag Rapid Test (CTK Biotech)] and three tests showed comparable performance [2019- nCoV Antigen test (Wondfo); Beijng Tigsun Diagnostics Co. Ltd (Tigsun); ACON biotech (Flowflex]. Sensitivity ranged between $22.2 \%$ and $88.9 \%$ for Omicron and $52.9 \%$ to $91.2 \%$ for Delta, confirming the high variability of sensitivity between the different tests.

The authors themselves point out the limitations of their work, to which special attention must be paid when interpreting the results: "The volume of viral transport medium added to the buffer was lower than what was recommended by some manufacturers, and for some Ag-RDTs there was no recommendation on the use of swab samples in VTM. Therefore, viral loads of the original sample and sensitivities observed in our sample collection cannot be compared to results obtained from clinical validations performed on fresh samples and our results should be interpreted as a comparison between Ag-RDTs and not as sensitivity thresholds for absolute viral loads and/or presence of infectious virus. Rather, we have investigated the lower end of sensitivity in the Ag-RDTs tested. Therefore, a reduced sensitivity in some tests, but not complete failure to detect Omicron could be of higher relevance in the beginning of the infection, when viral loads are still on the rise, and of less relevance once peak viral loads are reached."

The results of the study still need to be confirmed. Nevertheless, despite the limitations mentioned above, I consider the work to be highly valuable; the study contributes important new knowledge to science, and (although with caution) it can be used in practice. 
Considering possible publication in a peer-reviewed journal, a few points for correction should be noted:

- The part of the introduction, "In addition, a deletion (Del31-33) is found in the nucleocapsid of Omicron, as well as another mutation P13L" requires citation of reference.

- The study group was not described. While the lack of a detailed description of the study group can be explained by the fact that it is not particularly relevant in the context of these results, it should at least be written out of obligation how many people were included in the study - this information appears only in the discussion.

- There are 27 publications in the bibliography - 8 are websites, 11 are preprints, and only 8 are publications in peer-reviewed journals. I realise that this topic requires rapid publication. Perhaps the authors did not find any other available papers in peerreviewed journals, but this somewhat reduces the reliability of the content of the reviewed manuscript. 